\title{
Psittacara leucophthalmus (Aves: Psittacidae) como um novo hospedeiro de Paratanaisia bragai (Trematoda: Eucotylidae) no Brasil: achados clínicos e patológicos: relato de caso
}

[Psittacara leucophthalmus) as a new host of Paratanaisia bragai (Trematoda: Eucotylidae) in Brazil: clinical and pathological findings: case report]

T.G.W. Teodoro ${ }^{1}$, I.M. Oliveira Junior ${ }^{1}$, H.C. Lima $^{1}$, M.O. Reis ${ }^{1}$, J.L. Miranda ${ }^{1}$, S.M. Favoretto ${ }^{1}$, H.A. Pinto ${ }^{2}$, A.C.C. Lacreta Júnior ${ }^{1}$, A.T.B. Wouters ${ }^{1}$, M.S. Varaschin ${ }^{1 *}$

${ }^{1}$ Universidade Federal de Lavras - Lavras, MG

${ }^{2}$ Instituto de Ciências Biológicas - Universidade Federal de Minas Gerais - Belo Horizonte, MG

\section{RESUMO}

Paratanaisia bragai é um trematódeo que parasita rins e ureteres de aves domésticas e selvagens. Apesar de considerado pouco patogênico pode levar a complicações clínicas e morte em infecções severas. No presente estudo, um caso de parasitismo fatal em maritaca (Psittacara leucophthalmus) por P. bragai é relatado. A ave, oriunda da área urbana do município de Lavras, Minas Gerais, Brasil, teve manifestações clínicas de apatia, desidratação, dispneia e veio a óbito. Na necropsia foram observados rins aumentados de volume, pálidos, com superfície irregular e nodulações esbranquiçadas. Ao corte verificaram-se parasitos nos rins, que foram processados para montagem de lâminas permanentes e identificados segundo técnica de rotina como $P$. bragai. No exame histopatológico dos rins foram constatados infiltrado inflamatório linfoplasmocítico multifocal moderado, dilatação de túbulos e focos de regeneração tubular, associados a fibrose intersticial moderada no córtex renal. Na medula renal havia infiltrado de macrófagos, heterófilos, eosinófilos e células gigantes multinucleadas associado a parasitos em ductos coletores dilatados. O parasitismo de P. leucophthalmus por P. bragai é relatado pela primeira vez. A importância de se considerar este parasito entre as possibilidades diagnósticas em aves com insuficiência renal é brevemente discutida.

Palavras-chave: doenças parasitárias, trematódeos, psitacídeos

\begin{abstract}
Paratanaisia bragai is a trematode parasite of the kidneys and ureters of poultry and wild birds. Despite its low pathogenicity, this parasite can lead to several clinical complications and death in heavy infections. In the present study, a fatal case of parasitism by P. bragai in a specimen of the White-eyed Parakeet, Psittacara leucophthalmus, is reported. The bird, coming from the urban area of the county of Lavras, Minas Gerais, Brazil, was clinically evaluated and revealed apathy, dehydration, dyspnea, and death. The gross findings were pale and enlarged kidneys, which also had irregular surface with whitish nodulations. Parasites were observed on cutting surface of the renal parenchyma. They were processed for assembly of permanent slides and identified as P. bragai according to routine technique. The histologic findings were mild multifocal lymphoplasmacytic infiltrate, tubular dilatation and foci of tubular regeneration related to mild interstitial fibrosis in the renal cortex, and infiltration of macrophages, heterophils, eosinophils and multinucleated giant cells associated with metazoan parasites in the collecting ducts in renal medulla. The parasitism of $\mathrm{P}$. leucophthalmus by $\mathrm{P}$. bragai is reported for the first time. The importance of considering this parasite among the diagnostic possibilities in birds with renal insufficiency is briefly discussed.
\end{abstract}

Keywords: parasitic diseases, trematodes, psittacidae

Recebido em 21 de setembro de 2017

Aceito em 9 de fevereiro de 2018

*Autor para correspondência (corresponding author)

E-mail: msvaraschin@dmv.ufla.br 


\section{INTRODUÇÃO}

Paratanaisia bragai (Santos, 1934) é um trematódeo pertencente à família Eucotylidae, que parasita rins e ureteres de diversas espécies de aves domésticas e selvagens, sendo mais comumente encontrado em columbiformes (Santos, 1934; Brandolini e Amato, 2007; Unwin et al., 2013; Costa et al., 2015). No Brasil, há relatos de mais de 10 espécies de aves, incluindo galinhas, pombos, perus, galinhas d'Angola, araras e faisões, naturalmente infectadas em diferentes localidades do país (Fernandes et al., 2015), com vários casos relatados em espécies de psitacídeos (Tab. 1).

Tabela 1. Espécies de aves parasitadas por Paratanaisia bragai na literatura referenciada

\begin{tabular}{|c|c|c|c|}
\hline Espécie hospedeira & Origem & Caracterização da doença & Fonte \\
\hline Ara ararauna & $\begin{array}{c}\text { Criatório conservacionista, } \\
\text { Itatinga /SP }\end{array}$ & Doença fatal & Silva et al., 2016 \\
\hline Ara ararauna & $\begin{array}{c}\text { Vida livre, } \\
\text { Belo Horizonte/MG }\end{array}$ & Doença fatal & Luppi et al., 2007 \\
\hline Ara macao & $\begin{array}{l}\text { Cativeiro de centro de } \\
\text { reabilitação, } \\
\text { Costa Rica }\end{array}$ & Doença fatal & $\begin{array}{l}\text { Alfaro-Alarcón et } \\
\text { al., } 2015\end{array}$ \\
\hline $\begin{array}{c}\text { Columba inornata } \\
\text { wetmorei }\end{array}$ & $\begin{array}{l}\text { Cativeiro, } \\
\text { Porto Rico }\end{array}$ & Doença fatal & Arnizaut et al., 1992 \\
\hline Columba livia & $\begin{array}{l}\text { Vida livre, } \\
\text { Seropédica/RJ }\end{array}$ & $\begin{array}{c}\text { Doença subclínica; aves } \\
\text { submetidas a eutanásia para } \\
\text { estudo do parasito. }\end{array}$ & $\begin{array}{c}\text { Brandolini e Amato, } \\
2007\end{array}$ \\
\hline $\begin{array}{l}\text { Gallicolumba } \\
\text { crinigera }\end{array}$ & $\begin{array}{l}\text { Cativeiro, } \\
\text { Reino Unido }\end{array}$ & Doença subclínica & Unwin et al., 2013 \\
\hline $\begin{array}{l}\text { Gallicolumba } \\
\text { luzonica }\end{array}$ & $\begin{array}{l}\text { Cativeiro, } \\
\text { Reino Unido }\end{array}$ & Doença fatal & Unwin et al., 2013 \\
\hline $\begin{array}{l}\text { Gallicolumba } \\
\text { rufigula }\end{array}$ & $\begin{array}{l}\text { Cativeiro, } \\
\text { Reino Unido }\end{array}$ & Doença subclínica & Unwin et al., 2013 \\
\hline Paradisaea rubra & $\begin{array}{l}\text { Cativeiro, } \\
\text { Reino Unido }\end{array}$ & $\begin{array}{c}\text { Doença clínica com } \\
\text { eutanásia pelo mau } \\
\text { prognóstico }\end{array}$ & Unwin et al., 2013 \\
\hline Pavo cristatus & $\begin{array}{l}\text { Cativeiro, } \\
\text { Lavras/MG }\end{array}$ & Doença subclínica & Costa et al., 2015 \\
\hline Primolius maracana & $\begin{array}{c}\text { Cativeiro, } \\
\text { Belo Horizonte, } \mathrm{MG}\end{array}$ & Doença fatal & Luppi et al., 2007 \\
\hline $\begin{array}{l}\text { Ptilinopus } \\
\text { jambu }\end{array}$ & $\begin{array}{c}\text { Cativeiro, } \\
\text { Reino Unido }\end{array}$ & Doença subclínica & Unwin et al., 2013 \\
\hline $\begin{array}{c}\text { Pyrrhura } \\
\text { leucotis }\end{array}$ & $\begin{array}{c}\text { Cativeiro, } \\
\text { Belo Horizonte, } \mathrm{MG}\end{array}$ & Doença fatal & Luppi et al., 2007 \\
\hline $\begin{array}{l}\text { Stigmatopelia } \\
\text { senegalensis }\end{array}$ & $\begin{array}{c}\text { Cativeiro, } \\
\text { Reino Unido }\end{array}$ & Doença subclínica & Unwin et al., 2013 \\
\hline Zenaida graysoni & $\begin{array}{l}\text { Cativeiro, } \\
\text { Reino Unido }\end{array}$ & Doença fatal & Unwin et al., 2013 \\
\hline
\end{tabular}

As aves se infectam pela ingestão de moluscos gastrópodes terrestres (Subulina octona e Leptinaria unilamellata) contendo metacercárias infectantes, as quais atingem a forma adulta nos rins (Maldonado, 1945). O parasitismo por $P$. bragai está relacionado a alterações patológicas diversas, incluindo nefrite granulomatosa acentuada, que pode ser fatal (Luppi et al., 2007; Unwin et al., 2013; Silva et al., 2016).
Embora as aves da ordem Psittaciformes possam ser acometidas por uma grande variedade de parasitos, incluindo helmintos (Grespan e Raso, 2014), a infecção dessas aves por espécies de Paratanaisia foi relatada apenas nos últimos anos, havendo, inclusive, a associação desses trematódeos com a ocorrência de mortalidade nesse grupo de aves (Luppi et al., 2007; AlfaroAlarcón et al., 2015). Essas informações revelam 
que, apesar de P. bragai ser considerado pouco patogênico, sérias complicações clínicas podem ser observadas em infecções intensas, sendo variações na suscetibilidade dos animais à infecção e na formação de lesões possivelmente relacionadas à espécie e à idade dos animais infectados (Unwin et al., 2013). Nesse sentido, estudos visando avaliar a diversidade de hospedeiros e aspectos patológicos relacionados à interação $P$. bragai e aves Psittasiformes são ainda necessários.

No presente estudo, o parasitismo de maritacas (Psittacara leucophtalmus) por P. bragai é relatado pela primeira vez. São apresentados os achados patológicos associados a essa parasitose e discutidos os aspectos relacionados ao diagnóstico dessa parasitose em psitacídeos.

\section{CASUÍSTICA}

Um periquitão-maracanã (Psittacara leucophthalmus) fêmea foi atendido em uma clínica particular no município de Lavras, Minas Gerais, em maio de 2016. A ave estava com a tutora havia um ano, que a mantinha em viveiro em residência urbana, o qual ficava em área próxima à cozinha, no entanto, na maior parte do dia a ave ficava solta no jardim e na área de serviço da casa. A dieta era composta por sementes de girassol, frutas e bolachas. Ao exame clínico observou-se apatia, desidratação e dispneia severa. À auscultação foi constatado ruído semelhante à roce pleural acompanhando a frequência respiratória em região caudal da cavidade celomática, com suspeita inicial de aerossaculite severa. Foi realizada oxigenoterapia com auxílio de máscara, porém, durante o procedimento, a ave veio a óbito e foi encaminhada para necropsia no Setor de Patologia Veterinária da Universidade Federal de Lavras (UFLA), Minas Gerais.

Macroscopicamente observou-se depósito de material brancacento granular e opaco na superfície do fígado e do coração (Fig. 1).

Os rins estavam aumentados de volume, moderadamente pálidos e tinham superfície irregular com nodulações esbranquiçadas (Fig. 2).

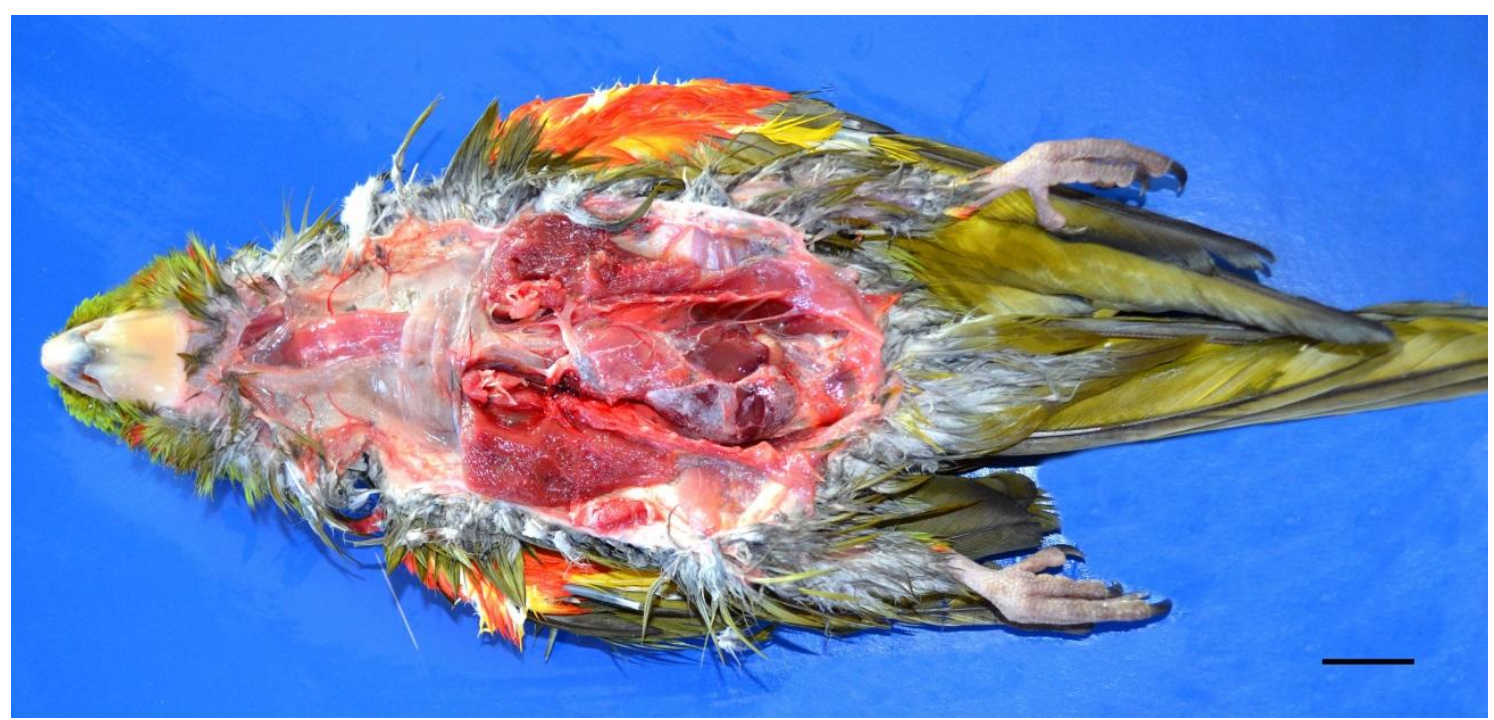

Figura 1. Achados de necropsia de Psittacara leucophthalmus com parasitismo renal por Paratanaisia bragai. Depósito de material brancacento granular e opaco em superfície hepática e cardíaca.

Escala: $3,0 \mathrm{~cm}$ 


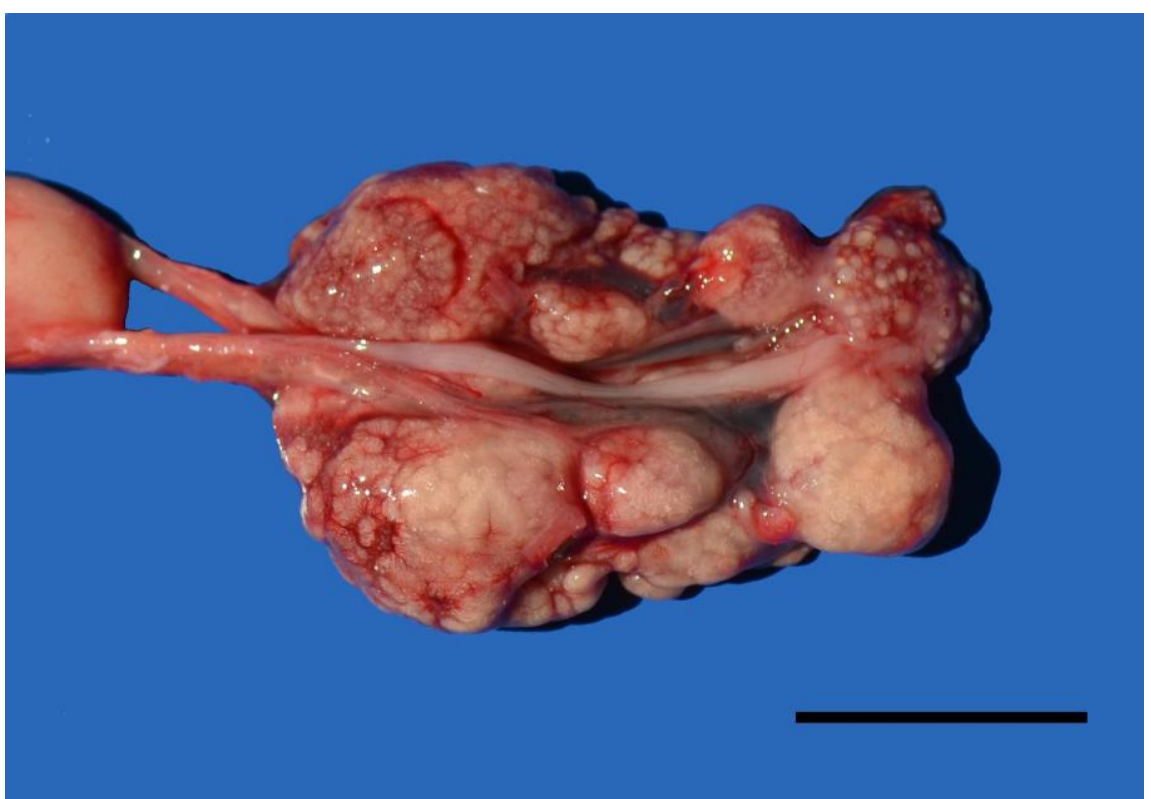

Figura 2. Achados de necropsia de Psittacara leucophthalmus com parasitismo renal por Paratanaisia bragai. Rins aumentados de volume, pálidos e com superfície irregular. Escala: $1 \mathrm{~cm}$

Ao corte, foram visualizadas estruturas alongadas brancacentas, de aproximadamente 0,3 $\mathrm{cm}$, entremeadas no parênquima renal. Amostras de vários tecidos foram coletadas e fixadas em formol $10 \%$ tamponado, processadas segundo técnica histológica de rotina, cortadas a $5 \mu \mathrm{m}$ e coradas com hematoxilina e eosina. Amostras das estruturas alongadas e brancacentas encontradas nos rins foram coletadas, fixadas em álcool $70 \%$ e encaminhadas para identificação taxonômica no Departamento de Parasitologia, Instituto de Ciências Biológicas da Universidade Federal de Minas Gerais (UFMG). Os parasitos foram submetidos à coloração por carmim acetoalúmen, seguida de desidratação em série de álcoois em concentração crescente, clarificação em creosoto de Faia e montagem entre lâmina e lamínula em bálsamo do Canadá. Alguns exemplares foram clarificados em lactofenol e montados em preparações não permanentes para o estudo das escamas cuticulares em microscópio óptico. A identificação taxonômica do parasito foi baseada em critérios morfológicos e morfométricos. A determinação do gênero foi baseada na chave de identificação proposta por Gibson et al. (2002). A determinação específica foi baseada na comparação com dados descritos por diferentes autores (Santos, 1934; Brandolini e Amato, 2007; Fernandes et al., 2015).
O estudo morfológico dos parasitos recuperados possibilitou a identificação de P. bragai. Entre os representantes da família Eucotylidae, o gênero Paratanaisia é caracterizado principalmente pela presença de vitelária ocupando as regiões pré e pós-testiculares (Gibson et al., 2002). Os parasitos não comprimidos (Fig. 3A) mediam 1428 (1009-2283) $\mu \mathrm{m}$ de comprimento por 395 (240-629) $\mu \mathrm{m}$ de largura. Foi observada ventosa oral subterminal medindo 155 (115-193) $\mu \mathrm{m} \mathrm{de}$ comprimento por 163 (106-221) $\mu \mathrm{m}$ de largura, seguida de faringe muscular medindo 61 (55-72) $\mu \mathrm{m}$ por 65 (57-78) $\mu \mathrm{m}$; esôfago curto; ventosa ventral e órgãos sexuais de difícil visualização devido à sobreposição com ovos. Estes eram acastanhados e mediam 38 (36-39) $\mu \mathrm{m}$ de comprimento por 16 (15-18) $\mu \mathrm{m}$ de largura. A vitelária era folicular, bilateral, ocupando a região entre o terço posterior do corpo, maior parte do corpo, e a bifurcação esofágica. Havia escamas tegumentares (Fig. 3B) medindo 8 (7-9) $\mu \mathrm{m}$ por 11 (10-12) $\mu \mathrm{m}$, com morfologia característica da que é conhecida para a espécie, como a descrita por Brandolini e Amato (2007). Entre as espécies do gênero relatadas na América do Sul, as que mais se assemelham a $P$. bragai é Paratanaisia confusa (Freitas, 1951), a qual tem tegumento com espinhos em vez de escamas; e Paratanaisia robusta (Freitas, 1951), que difere de $P$. bragai pelo formato das escamas tegumentares. 


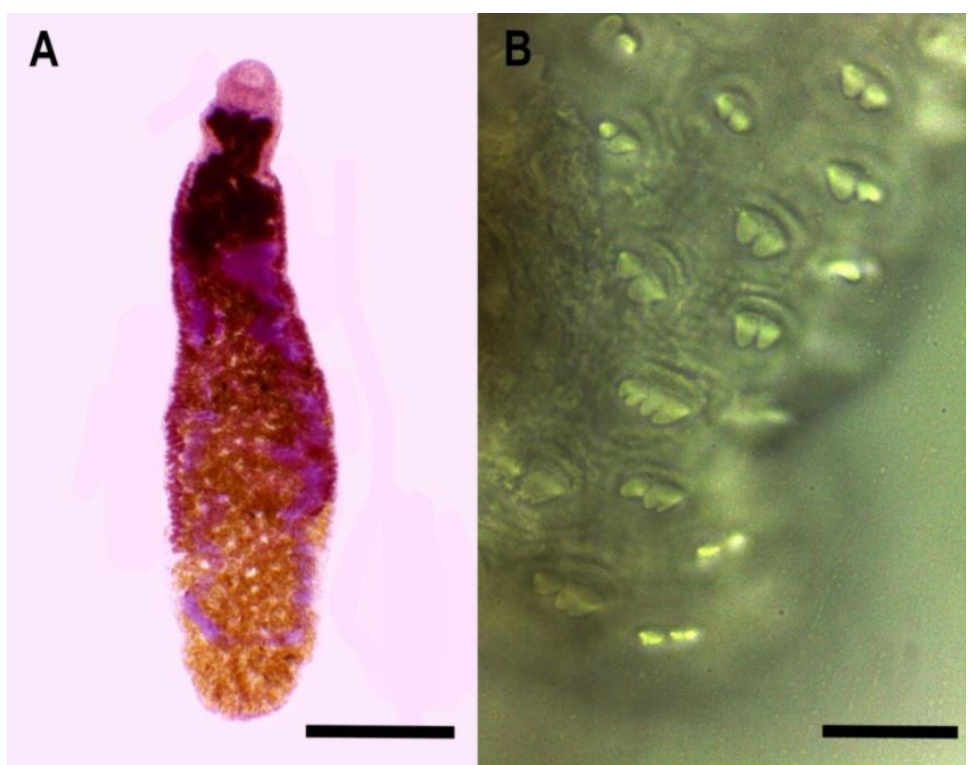

Figura 3. Paratanaisia bragai encontrado em rim de Psittacara leucophthalmus. (A) Parasito recuperado do rim (coloração carmim acetoalúmen). (B) Detalhe das escamas tegumentares em microscopia óptica. Escalas: $\mathrm{A}=500 \mu \mathrm{m} ; \mathrm{B}=20 \mu \mathrm{m}$.

O exame histopatológico do rim revelou infiltrado inflamatório linfoplasmocítico multifocal moderado em córtex e medula renal, dilatação tubular e focos de regeneração tubular, associados a fibrose intersticial moderada. Infiltrado de macrófagos, heterófilos, eosinófilos e células gigantes multinucleadas, associado a parasitos em ductos coletores, foi observado na medula renal (Fig. 4). Nos cortes histológicos foram visualizadas seções do parasito adulto, caracterizadas por estruturas acelomadas com ventosa oral, revestidas por tegumento com escama, e, no seu interior, foram visualizados ovos embrionados operculados e de casca amarronzada, além de testículos (Fig. 5).

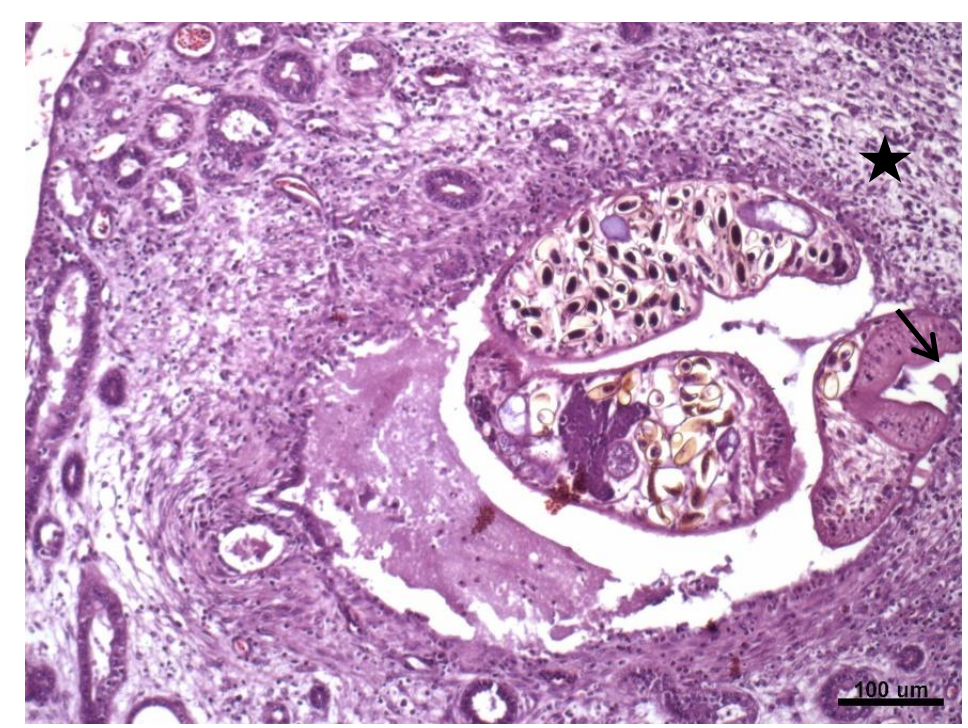

Figura 4. Achados histológicos em rim de Psittacara leucophthalmus com parasitismo por Paratanaisia bragai. Infiltrado inflamatório multifocal moderado de linfócitos, plasmócitos e heterofilos (estrela) em medula renal. Notar seções do parasito em ducto coletor dilatado, com ventosa anterior evidente (seta). HE. Escala: $100 \mu \mathrm{m}$. 


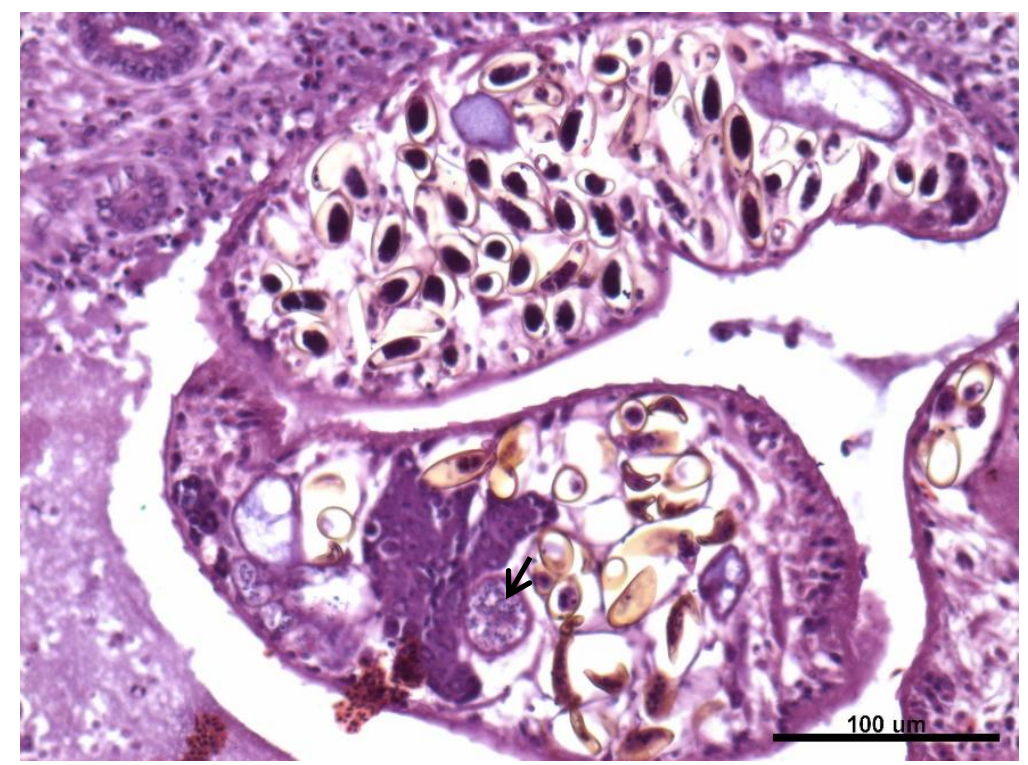

Figura 5. Detalhes histológicos de Paratanaisia bragai no rim de Psittacara leucophthalmus. As seções do trematódeo são revestidas por tegumento com escamas (saliências densas e eosinofílicas no tegumento) e são visualizados ovos embrionados operculados de casca amarronzada e testículo (seta) no interior do corpo do parasito. H.E. Escala: $100 \mu \mathrm{m}$

\section{DISCUSSÃO}

Os achados macroscópicos no exemplar de $P$. leucophthalmus avaliado: rins aumentados de volume, pálidos, com superfície irregular e nodulações esbranquiçadas, associados aos helmintos, são semelhantes aos achados relatados por outros autores, inclusive em espécies de Psittaciformes (Luppi et al., 2007; Alfaro-Arcón et al., 2015, Silva et al., 2016). As alterações histológicas observadas nos rins foram semelhantes às descritas em casos de parasitismo por Paratanaisia sp. em outras espécies de aves (Silva et al., 2016).

A ocorrência de $P$. bragai é relatada pela primeira vez em $P$. leucophthalmus. A parasitose não é comumente encontrada em aves da ordem Psittaciformes, provavelmente por serem animais arbóreos, o que diminui as chances de ingestão de moluscos gastrópodes contendo formas infectantes. $\mathrm{O}$ registro desse parasito em psitacídeos é relativamente raro e recente. O primeiro caso foi relatado em tiriba-de-orelhabranca (Pyrrhura leucotis), maracanã-verdadeira (Propyrrhura maracanã) e arara-canindé (Ara ararauna), em Belo Horizonte, MG (Luppi et al., 2007). Mais recentemente, o parasito foi encontrado também em A. ararauna, no estado de São Paulo (Silva et al., 2016), e em arara- macau (Ara macao), na Costa Rica (AlfaroAlarcón et al., 2015). Em todos esses casos, as aves eram mantidas em cativeiro, criadouros, zoológicos ou centros de resgate de animais, havia pelo menos três anos. Nesse sentido, é possível que casos de paratanaisiose diagnosticados em psitasídeos resultem da ocorrência de "spillover" entre os parasitos encontrados, principalmente de columbiformes no ambiente urbano, transmitidos a psitacídeos mantidos fora de seu habitat natural, pela ingestão de moluscos terrestres naturalmente infectados. De fato, Alfaro-Alarcón et al. (2015) correlacionam casos atendidos ao período de chuvas e ao avistamento das aves alimentando-se de invertebrados no chão e nas árvores, o que reforça a possibilidade de ocorrência do fenômeno anteriormente descrito.

O diagnóstico ante mortem da infecção de aves por P. bragai, e mesmo por outros membros da família Eucotylidae, não tem sido realizado, sendo a maioria dos casos relatados baseada em achados de necropsia. Nesses casos, o diagnóstico definitivo é obtido pelo exame histopatológico dos rins e da coleta, avaliação microscópica de exemplares do parasito e identificação taxonômica. Embora a identificação parasitológica pelo achado de ovos nas fezes de aves seja possível, tal abordagem 
não tem sido rotineiramente utilizada. O parasitismo por $P$. bragai pode levar a alterações na função renal. As alterações compatíveis com gota visceral observadas na serosa hepática corroboram alteração renal. Dieta rica em proteínas também é considerada condição favorável para a ocorrência de gota, mas a ave examinada tinha uma dieta pobre em proteínas, o que aumentava as evidências para a associação das alterações de gota úrica com as lesões renais pelo parasitismo. A lesão renal causada pela infecção por $P$. bragai é relacionada à fricção das escamas tegumentares na parede de túbulos renais (Costa et al., 2015), a qual se torna mais acentuada nos casos de elevada carga parasitária (Unwin et al., 2013, Silva et al., 2016). A morte de psitacídeos por insuficiência renal crônica secundária a nefrite granulomatosa por Paratanaisia sp. foi relacionada a casos relatados no Brasil e na Costa Rica (Luppi et al., 2007; Alfaro-Alarcón et al., 2015). A dilatação de túbulos renais e ductos coletores foi observada e tem sido atribuída ao parasitismo pelo trematódeo (Alfaro-Alarcón et al., 2015). Como os rins têm capacidade regenerativa limitada, o parasitismo pode cursar com alta morbidade (Unwin et al., 2013).

A alteração à ausculta respiratória salienta a importância do diagnóstico diferencial entre gota úrica visceral e aerossaculite quando da ausculta de roce pleural em região caudal de cavidade celomática. Em um primeiro momento, devido à maior ocorrência de problemas respiratórios em aves de companhia, a aerossaculite é o primeiro diagnóstico clínico sugerido. No entanto, com os crescentes relatos de parasitismo por Paratanaisia sp., este deve ser considerado um importante diagnóstico diferencial. O parasitismo renal por Paratanaisia sp. deve ser considerado no diagnóstico de psitaciformes com sinais de insuficiência renal. Salienta-se a relevância de exames bioquímicos de sangue associados ao exame de fezes no atendimento de aves cativas, os quais podem auxiliar no diagnóstico de insuficiência renal resultante da infecção por $P$. bragai. Tratamentos à base de fenbendazol e praziquantel já foram preconizados para columbiformes e passeriformes (Arnizaut et al., 1992; Unwin et al., 2013), porém ainda são escassos os estudos envolvendo a abordagem terapêutica em psitaciformes infectados por esse parasito.
A gravidade das alterações renais de nefrite piogranulomatosa descritas neste trabalho contribui para o conhecimento da patogenicidade de Paratanaisia sp. em psitacídeos.

\section{CONCLUSÃO}

Psittacara leucophthalmus é descrito como um novo hospedeiro do trematódeo renal Paratanaisia bragai. O parasitismo está associado à ocorrência de nefrite crônica.

\section{AGRADECIMENTO}

À Fundação de Amparo à Pesquisa do Estado de Minas Gerais - FAPEMIG, pelo auxílio financeiro (Processo CVZ-PPM 00763-16).

\section{REFERÊNCIAS}

ALFARO-ALARCÓN, A.; MORALES, J.A.; VENEZIANO, V. et al. Fatal Paratanaisia bragai (Digenea:Eucotylidae) infection in Scarlet macaws (Ara macao) in Costa Rica. Acta Parasitol., v.60, p.548-552, 2015.

ARNIZAUT, A.B.; HAYES, L.; OLSEN, G.H. et al. An epizootic of Tanaisia bragai in a captive population of Puerto Rican plain pigeon (Columba inornata wetmorei). Ann. N.Y. Acad. Sci., v.653, p.202-205, 1992.

BRANDOLINI, S.V.P.B.; AMATO, S.B. Morfologia externa de espécimes adultos de Paratanaisia bragai (Santos, 1934) (Digenea: Eucotylidae). Rev. Bras. Parasitol. Vet., v.16, p.129-132, 2007.

COSTA, R.C.; AMBRÓSIO, N.A.; SOARES, B.A. et al. Pathological and parasitological aspects of the peacock (Pavo cristatus) infection by Paratanaisia bragai. Pesqui. Vet. Bras., v.35, p.466-469, 2015.

FERNANDES, B.M.M.; JUSTO, M.C.N.; CARDENAS, M.Q. et al. South American trematodes parasites of birds and mammals. Rio de Janeiro: Oficina de Livros, 2015. 516p.

FREITAS J.F.T. Revisão da família Eucotylidae Skrjabin, 1924 (Trematoda). Mem. Inst. Oswaldo Cruz, v.49, p.33-271, 1951.

GIBSON, D.I.; JONES, A.; BRAY, R.A. Keys to the trematoda. Wallingford, UK: CABI Publishing, 2002. v.1, 544p. 
GRESPAN, A.; RASO, T.F. Psittaciformes (araras, papagaios, periquitos, calopsitas e cacatuas). In: CUBAS, Z.S.; SILVA, J.C.R.; CATÃO-DIAS, J.L. Tratado de animais selvagens. 2.ed. São Paulo: Roca, 2014. v.1, p.586-587.

LUPPI, M.M.; MELO, A.L.; MOTTA, R.O.C. et al. Granulomatous nephritis is psittacines associated with parasitism by the trematode Paratanaisia spp. Vet. Parasitol., v.146, p.363366, 2007.

MALDONADO, J.F. The life cycle of Tarmelania bragai, Santos 1934 (Eucotylidae), kidney fluke of the domestic pigeons. $J$. Parasitol., v.31, p.306-314, 1945.
SANTOS, V. Monostomose renal das aves domésticas. Rev. Dep. Nac. Prod. Anim., v.1, p.203-215, 1934.

SILVA, T.M.; PAVAN, L.F.; GUIMARÃESOKAMOTO, P.T.C. et al. First record of Paratanaisia bragai (Digenea: Eucotylidae) in blue and gold macaw (Ara ararauna). Braz. J. Vet. Parasitol., v.25, p.112-115, 2016.

TUNWIN, S.; CHANTREY, J.; CHATTERTON, J. et al. Renal trematode infection due to Paratanaisia bragai in zoo housed Columbiformes and a rea bird-of-paradise (Paradaise rubra). Intern. J. Parasitol. Parasites Wildl., v.2, p.32-41, 2013. 